\title{
On minimal representations by a family of sublinear functions
}

\author{
Jerzy Grzybowski • Mahide Küçük • Yalçın Küçük • \\ Ryszard Urbański
}

Received: 30 December 2013 / Accepted: 14 February 2014 / Published online: 5 March 2014

(C) The Author(s) 2014. This article is published with open access at Springerlink.com

\begin{abstract}
This paper is a continuation of Grzybowski et al. (J Glob Optim 46:589-601, 2010) and is motivated by the study of exhausters i.e. families of closed convex sets. By Minkowski duality closed convex sets correspond to sublinear functions. Here we study the criteria of reducing representations of pointwise infimum of an infinite family of sublinear functions. A family $\left\{f_{i}\right\}_{i \in I}$ of sublinear functions is by definition an exhaustive family of upper convex approximations of its pointwise infimum $\inf _{i \in I} f_{i}$. A family of closed convex sets is by definition an exhauster of a pointwise infimum of a family of support functions of these convex sets. We establish codependence between infimum of a subset of MinkowskiRådström-Hörmander cone and translation property of intersection of an exhauster (Sect. 4 ), between reducing an exhauster to single convex set and shadowing property of intersection of an exhauster (Theorem 5.1) and among all four of these properties (Theorem 6.1). In Grzybowski et al. (2010) the first example of two different minimal upper exhausters of the same function was presented. Here we give an example of infinitely many minimal exhausters of the same $p h$-function (Example 7.2). In Sect. 8, we give a criterion of minimality of an exhauster with the help of polars of sets belonging to the exhauster. We illustrate this criterion with two interesting examples (Example 8.3).
\end{abstract}

Support of Anadolu University. Project no. 1201F018.

J. Grzybowski $(\bowtie) \cdot$ R. Urbański

Faculty of Mathematics and Computer Science, Adam Mickiewicz University,

Umultowska 87, 61-614 Poznań, Poland

e-mail: jgrz@amu.edu.pl

R. Urbański

e-mail: rich@amu.edu.pl

M. Küçük · Y. Küçük

Faculty of Science, Department of Mathematics, Anadolu University,

Yunus Emre Campus, 26470 Eskişehir, Turkey

e-mail: mkucuk@anadolu.edu.tr

Y. Küçük

e-mail: ykucuk@anadolu.edu.tr 
Keywords Convex sets · Sublinear functions · Minkowski duality · Upper/lower exhauster · Minkowski-Rådström-Hörmander cone · Translation property of intersection · Shadowing of a family of sets · Polar of convex set

Mathematics Subject Classification $46 \mathrm{~B} 20 \cdot 52 \mathrm{~A} 05 \cdot 54 \mathrm{~B} 20$

\section{Introduction}

The main object of this paper is to study reducibility and minimality of exhausters. The notion of upper/lower exhauster was introduced by Demyanov and Rubinov [10]. Exhausters were studied in a series of papers (see for example [3-6,28,29]).

Directional derivative of a function $f: X \longrightarrow \mathbb{R}$ at $x_{0} \in X$ is a positively homogenous function $X \ni v \mapsto f^{\prime}\left(x_{0} ; v\right)=\lim _{t \rightarrow 0^{+}} \frac{f\left(x_{0}+t v\right)-f\left(x_{0}\right)}{t}$. If the directional derivative $f^{\prime}\left(x_{0} ; \cdot\right)$ is linear then $f$ is differentiable at $x_{0}$. If a function $f$ is convex then $f^{\prime}\left(x_{0} ; \cdot\right)$ is sublinear. If the function $f^{\prime}\left(x_{0} ; \cdot\right)$ is sublinear then $f$ is called subsmooth [27]. If a function $f$ is a $d c$-function (difference of convex functions) then $f^{\prime}\left(x_{0} ; \cdot\right)$ a $d s$-function (difference of sublinear functions). If the function $f^{\prime}\left(x_{0} ; \cdot\right)$ is a $d s$-function then $f$ is called quasi differentiable (tangentially $d c$-function) at $x_{0}([7,10,23])$.

Let us notice that each $d s$-function $f$ can be represented by a pair $(A, B)$ of bounded closed convex sets, where $f$ is equal to the difference of two support functions $p_{A}-p_{B}$. However, a much broader class of positively homogenous functions can be represented by a family of bounded closed convex sets as $f=\inf _{i \in I} p_{A_{i}}$. For example a very natural function $f\left(x_{1}, x_{2}\right)=\sqrt{\left|x_{1} x_{2}\right|}$ is not a $d s$-function but it is a pointwise infimum of support functions of infinitely many rectangles [12].

The Minkowski duality is the correspondence between subdifferentials and support functions. Let $\operatorname{Sublin}\left(\mathbb{R}^{n}\right)$ be the set of all sublinear functions and $\mathcal{B}\left(\mathbb{R}^{n}\right)$ be the family of all nonempty bounded closed convex sets in $\mathbb{R}^{n}$. The set $\left.\partial p\right|_{0}=\{x \mid\langle x, \cdot\rangle \leqslant$ $p\} \in \mathcal{B}\left(\mathbb{R}^{n}\right)$ is called the subdifferential of $p \in \operatorname{Sublin}\left(\mathbb{R}^{n}\right)$ at 0 . The function $p_{A}=$ $\sup _{x \in A}\langle x, \cdot\rangle \in \operatorname{Sublin}\left(\mathbb{R}^{n}\right)$ is called the support function of the set $A \in \mathcal{B}\left(\mathbb{R}^{n}\right)$. The correspondence between (Sublin $\left.\left(\mathbb{R}^{n}\right),+, \cdot, \leqslant\right)$ and $\left(\mathcal{B}\left(\mathbb{R}^{n}\right),+, \cdot, \subset\right.$ ) is an isomorphism of ordered abstract convex cones. The Minkowski duality can be extended to the family $\mathcal{C}\left(\mathbb{R}^{n}\right)$ of all nonempty closed convex sets on one hand and to the set $\operatorname{Sublin}^{*}\left(\mathbb{R}^{n}\right)$ of all sublinear functions taking values in $\overline{\mathbb{R}}=\mathbb{R} \cup\{\infty\}$ on the other hand. Here, however, the addition $A+B=\{a+b \mid a \in a, b \in B\}$ has to be replaced with $A \dot{+} B=$ $\overline{A+B}$.

The generalized Minkowski duality is the correspondence between the set $D S\left(\mathbb{R}^{n}\right)$ of differences of sublinear functions and the quotient space $\widetilde{\mathbb{R}^{n}}=\mathcal{B}^{2}\left(\mathbb{R}^{n}\right) / \sim$, where $(A, B) \sim$ $(C, D)$ if and only if $A+D=B+C$. For $f=p-q \in D S\left(\mathbb{R}^{n}\right)$ the quasi differential of $f$ at 0 is the pair of sets $\left(\left.\partial p\right|_{0},\left.\partial q\right|_{0}\right)$ or, better, a quotient class $\left[\left(\left.\partial p\right|_{0},\left.\partial q\right|_{0}\right)\right] \in \widetilde{\mathbb{R}^{n}}$. On the other hand the difference $p_{A}-p_{B} \in D S\left(\mathbb{R}^{n}\right)$ corresponds to $[(A, B)] \in \mathbb{R}^{n}$.

The family of all nonempty bounded closed convex sets with addition and multiplication $\left(\mathcal{B}\left(\mathbb{R}^{n}\right),+, \cdot\right)$ is an abstract convex cone in the following sense. Let $A, B, C \in \mathcal{B}(X), \alpha, \beta \in$ $\mathbb{R}_{+}$. We have $A+B=\{a+b \mid a \in A, b \in B\},(A+B)+C=A+(B+C), A+B=B+A$, $A+\{0\}=A,(\alpha \beta) A=\alpha(\beta A), 1 A=A, \alpha(A+B)=\alpha A+\alpha B$ and $(\alpha+\beta) A=\alpha A+\beta A$. The family of all nonempty closed convex sets with addition and multiplication $\left(\mathcal{C}\left(\mathbb{R}^{n}\right), \dot{+}, \cdot\right)$ is an abstract convex cone in exactly the same way. However, the addition is modified, i.e. $A \dot{+} B=\overline{A+B}$. 
The quotient set $\widetilde{\mathbb{R}^{n}}=\mathcal{B}^{2}\left(\mathbb{R}^{n}\right) / \sim$, where $(A, B) \sim(C, D)$ if and only if $A+D=B+C$, is a vector space called Minkowski-Rådström-Hörmander (MRH) space with the following operations of addition and multiplication by scalars. Let $A, B, C, D \in \mathcal{B}\left(\mathbb{R}^{n}\right), \alpha \in \mathbb{R}$, $\tilde{x}=[A, B]=[(A, B)]$ and $\tilde{y}=[C, D]$. We have $\tilde{x}+\tilde{y}=[A, B]+[C, D]=[A+C, B+D]$ and $\alpha \tilde{x}=\alpha[A, B]=\left[\alpha^{+} A+\alpha^{-} B, \alpha^{-} A+\alpha^{+} B\right]$, where $\alpha^{+}=\max (\alpha, 0)$ and $\alpha^{-}=(-\alpha)^{+}$. Then $-\tilde{x}=[B, A]$ and $(-1) \tilde{x}=[B, A]=-\tilde{x}$.

The MRH space with the ordering defined by $\tilde{x} \leqslant \tilde{y}$ if and only if $A+D \subset B+C$ is a lattice with $\sup (\tilde{x}, \tilde{y})=[(A+D) \vee(B+C), B+D]$ and $\inf (\tilde{x}, \tilde{y})=[A+C,(A+D) \vee(B+C)]$, where $A \vee B=\overline{\operatorname{conv}}(A \cup B)$. The MRH space may be called a vector space of convex sets $\tilde{x}=[A, B]=\overrightarrow{B A}$, where the vector $\overrightarrow{B A}$ has the initial convex set $B$ and the terminal set $A$. We can think of the elements of MRH space as of virtual convex sets. Since the general subdifferential $\partial_{G}\left(p_{A}-p_{B}\right)$ is equal to the Minkowski difference $A-B=\{x \mid x+B \subset A\}$ and the Clarke subdifferential $\partial_{C l}\left(p_{A}-p_{B}\right)$ is equal to the Demyanov difference $A-B$, we may think that the virtual set $[A, B]$ contains $A-B$ and is contained in $A-B$. The MRH space was studied by many authors, e.g. Rådström [26], Hörmander [16], Rabinovich [25], Praksash, Sertel [22], Drewnowski [11], Urbański [31], Schmidt [30], Pallaschke, Scholtes, Urbański [19,20], Grzybowski [13], Markov [18] and Caprari, Penot [1].

The following laws enable us to embed the abstract convex cone $\mathcal{B}\left(\mathbb{R}^{n}\right)$ into the MRH space $\widetilde{\mathbb{R}^{n}}$.

Theorem 1.1 (Order law of cancellation) Let $A, B, C \subset \mathbb{R}^{n}$, B be bounded and $C \in \mathcal{C}\left(\mathbb{R}^{n}\right)$. Then $A+B \subset \overline{B+C}$ implies $A \subset C$.

The next law is a simple corollary.

Theorem 1.2 (Law of cancellation) Let $A, C \in \mathcal{C}\left(\mathbb{R}^{n}\right), B \in \mathcal{B}\left(\mathbb{R}^{n}\right)$. Then $A+B=C+B$ implies $A=C$.

The abstract convex cone $\mathcal{C}\left(\mathbb{R}^{n}\right)$ cannot be embedded into a vector space. It can be embedded into the following ordered MRH cone $\widetilde{\mathbb{R}_{c}^{n}}=\mathcal{C}\left(\mathbb{R}^{n}\right) \times \mathcal{B}\left(\mathbb{R}^{n}\right) / \sim$ (see [14]). For $A, C \in \mathcal{C}\left(\mathbb{R}^{n}\right), B, D \in \mathcal{B}\left(\mathbb{R}^{n}\right)$ we have $(A, B) \sim(C, D)$ if and only if $A+D=B+C$. The element $\tilde{x}=[A, B] \in \mathcal{C}\left(\mathbb{R}^{n}\right) \times \mathcal{B}\left(\mathbb{R}^{n}\right) / \sim$ is the quotient class of $(A, B)$. The sum is defined by $\tilde{x}+\tilde{y}=[A, B]+[C, D]=[A+C, B+D]$. The opposite element $-\tilde{x}=[B, A]$ exists only if $A \in \mathcal{B}\left(\mathbb{R}^{n}\right)$. Also, for $\alpha \geqslant 0$ we have $\alpha \tilde{x}=\alpha[A, B]=[\alpha A, \alpha B]$. The multiplication $(-1) \tilde{x}$ is not defined.

The MRH cone $\widetilde{\mathbb{R}_{c}^{n}}$ is an ordered cone. We have $\tilde{x} \leqslant \tilde{y}$ if and only if $A+D \subset B+C$. Also, $\sup (\tilde{x}, \tilde{y})=[(A+D) \vee(B+C), B+D]$. The infimum $\inf (\tilde{x}, \tilde{y})$ in general does not exist, hence the MRH cone $\widetilde{\mathbb{R}_{c}^{n}}$ is not a lattice.

\section{Upper exhausters of positively homogenous functions}

Some directional derivatives ( $p h$-functions) are not differences of two support functions. If a $p h$-function $h$ is upper semicontinuous then $h=\inf _{i \in I} p_{A_{i}}$ for some family $\left\{A_{i}\right\}_{i \in I} \subset$ $\mathcal{B}\left(\mathbb{R}^{n}\right)$. We denote $E^{*}(h)=\left\{A_{i}\right\}_{i \in I}$ and call $E^{*}(h)$ an upper exhauster of ph-function $h$. In a similar way $E^{*}(h)=\left\{B_{j}\right\}_{j \in J}$ is a lower exhauster of $h$ if $h=\sup _{j \in J} p_{B_{j}}$. The upper (lower) exhauster is not unique. Pshenichnyi [23] introduced upper convex approximation with sublinear functions greater than $h$. Demyanov and Rubinov $[8,9]$ studied exhaustive families of uca's. 
Let us notice that all $d s$-functions are represented by upper exhausters. We have $h=$ $p_{A}-p_{B}=\inf _{b \in B} p_{A-b}$. Hence $E^{*}=\{A-b\}_{b \in B}$ is an upper exhauster of $h$. Some functions represented with upper exhausters are not $d s$-functions. For example, the function $h\left(x_{1}, x_{2}\right)=\sqrt{\left|x_{1} \cdot x_{2}\right|}$, which is not a $d s$-function and has an upper exhauster $E^{*}(h)=$ $\left\{[-a, a] \times\left[-\frac{1}{4 a}, \frac{1}{4 a}\right]\right\}_{a>0}[12]$.

\section{Reducing finite exhausters}

In this section we gather results from [15] on reducing finite upper exhausters to single set in the form of Theorem 3.1 In such a case we have an exhauster of (Clarke) regular function $f=\inf _{i \in I} p_{A_{i}}$. It means that Frechet and Clarke (Michel-Penot) subdifferentials of $f$ coincide.

Theorem 3.1 (on reducing finite upper exhausters to single set) Let the set of indices $I$ be finite, $\left\{A_{i}\right\}_{i \in I} \subset \mathcal{B}\left(\mathbb{R}^{n}\right)$ and $A=\bigcap_{i \in I} A_{i}$. Then the following statements are equivalent:

(a) $\inf _{i \in I} p_{A_{i}}=p_{A}$.

(b) For all $\left\{a_{i}\right\} \in \prod_{i \in I} A_{i}$ we have $A \cap \bigvee_{i \in I} a_{i} \neq \emptyset$ (shadowing property).

(c) $\inf _{i \in I}\left[A_{i},\{0\}\right]=[A,\{0\}]$ (infimum in MRH space).

(d) For all $D \in \mathcal{B}\left(\mathbb{R}^{n}\right)$ we have $\bigcap_{i \in I}\left(D+A_{i}\right)=D+A$ (translation property of the intersection).

(e) $\bigvee_{i \in I} \sum_{k \in I \backslash\{i\}} A_{k}$ is a summand of $\sum_{i \in I} A_{i}$.

(f) $\sum_{i \in I} A_{i} \subset A+\bigvee_{i \in I} \sum_{k \in I \backslash\{i\}} A_{k}$.

Proof By Lemma 2.2 in [15] the set $\sup _{i \in I} \sum_{k \in I \backslash\{i\}} A_{k}$ is a summand of $\sum_{i \in I} A_{i}$ if and only if $\inf _{i \in I}\left[A_{i},\{0\}\right]=\left[\inf _{i \in I} A_{i},\{0\}\right]$. Since the supremum of convex sets $\sup _{i \in I} \sum_{k \in I \backslash\{i\}} A_{k}$ is the convex hull $\bigvee_{i \in I} \sum_{k \in I \backslash\{i\}} A_{k}$ and the infimum $\inf _{i \in I} A_{i}$ is the intersection $\bigcap_{i \in I} A_{i}$, the equivalence $(\mathrm{c}) \Leftrightarrow(\mathrm{e})$ follows immediately.

The equivalence (b) $\Leftrightarrow$ (c) follows from Theorem 5.2 in [15]. It should be mentioned, however, that in [15] the property (c) is called a shadowing property.

The equivalence (e) $\Leftrightarrow$ (f) is obvious. By summand, we understand a summand with respect to Minkowski addition. By the generalized Minkowski duality [20] we have $\inf _{i \in I} p_{A_{i}}=$ $p_{C}-p_{D}$ if and only if $\inf _{i \in I}\left[A_{i},\{0\}\right]=[C, D]$. Applying Theorem 1.7 in [15] the condition (a) appears equivalent to the equality $\left[\sum_{i \in I} A_{i}, \bigvee_{i \in I} \sum_{k \in I \backslash\{i\}} A_{k}\right]=[A,\{0\}]$, then to the equality

$$
\sum_{i \in I} A_{i}=A+\bigvee_{i \in I} \sum_{k \in I \backslash\{i\}} A_{k}
$$

and, at last, to (f).

(c) $\Rightarrow$ (d) Obviously, for any $D \in \mathcal{B}\left(\mathbb{R}^{n}\right)$ we have $D+A \subset \bigcap_{i \in I}\left(D+A_{i}\right)$. Let us denote $C=\bigcap_{i \in I}\left(D+A_{i}\right)$. For all $i \in I$ we have $C \subset D+A_{i}$. Hence $[C, D] \leqslant\left[A_{i},\{0\}\right]$. By the condition (c) we obtain $[C, D] \leqslant[A,\{0\}]$, and $C \subset D+A$.

(d) $\Rightarrow$ (c) Obviously, $[A,\{0\}] \leqslant\left[A_{i},\{0\}\right]$ for all $i \in I$. Let $[C, D] \leqslant\left[A_{i},\{0\}\right]$ for all $i \in I$. Then $C \subset D+A_{i}$. Hence $C \subset \bigcap_{i \in I}\left(D+A_{i}\right)$. By the condition (c) we get $C \subset D+A$. Then $[C, D] \leqslant[A,\{0\}]$.

The translation property (d) is new and we will elaborate it in the next sections. 
Fig. 1 The family of segments $\left\{A_{t}\right\}_{t \in \mathbb{R}}$ in Example 4.4

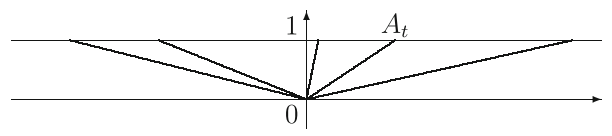

\section{Translation property of intersection}

Let us recall that for $A, B \in \mathcal{B}(\mathbb{R})$ the Minkowski (Hukuhara, Pontryagin) difference ([17, 21]) of $A$ and $B$ is the set $A-B=\{x \mid x+B \subset A\}$. The following property gives the equivalence between generalized properties (c) and (d) from Theorem 3.1.

Theorem 4.1 Let $\left\{\left(A_{i}, B_{i}\right)\right\}_{i \in I} \subset \mathcal{C}\left(\mathbb{R}^{n}\right) \times \mathcal{B}\left(\mathbb{R}^{n}\right), B \in \mathcal{B}\left(\mathbb{R}^{n}\right)$ and $A=\bigcap_{i \in I}\left(B+A_{i} \dot{-} B_{i}\right)$. Then the following statements are equivalent:

(a) $\inf _{i \in I}\left[A_{i}, B_{i}\right]=[A, B]$ (infimum in MRH cone).

(b) $\bigcap_{i \in I}\left(D+B+A_{i} \dot{-} B_{i}\right)=D+A$ for all $D \in \mathcal{B}\left(\mathbb{R}^{n}\right)$ (generalized translation property of intersection with respect to $\mathcal{B}\left(\mathbb{R}^{n}\right)$ ).

Proof (a) $\Rightarrow$ (b) Fix any $D \in \mathcal{B}\left(\mathbb{R}^{n}\right)$. Obviously, $D+A \subset \bigcap_{i \in I}\left(D+B+A_{i} \dot{-} B_{i}\right)$. Hence $C=\bigcap_{i \in I}\left(D+B+A_{i} \dot{-} B_{i}\right) \neq \emptyset$, and we have $C \subset D+B+A_{i} \dot{-} B_{i}$. Therefore $C+B_{i} \subset$ $D+B+A_{i}$, and $[C, D+B] \leqslant\left[A_{i}, B_{i}\right]$. Now by (a) we get $[C, D+B] \leqslant[A, B]$. Hence $C+B \subset D+B+A$, and by the order law of cancelation we have $C \subset D+A$.

(b) $\Rightarrow$ (a) From (b) we have $A=\bigcap_{i \in I}\left(B+A_{i} \dot{-} B_{i}\right)$. Hence $A \subset B+A_{i} \dot{-} B_{i}$, and $[A, B] \leqslant\left[A_{i}, B_{i}\right]$ for all $i \in I$. Now given $[C, D] \leqslant\left[A_{i}, B_{i}\right]$ this is equivalent to $C+B_{i} \subset$ $D+A_{i}$. Hence $C+B+B_{i} \subset D+B+A_{i}$, and $C+B \subset D+B+A_{i} \dot{-} B_{i}$ for all $i \in I$. Therefore $C+B \subset \bigcap_{i \in I}\left(D+B+A_{i} \dot{-} B_{i}\right)$. By (b) we have $C+B \subset D+A$, hence $[C, D] \leqslant[A, B]$.

Corollary 4.2 Let $\left\{A_{i}\right\}_{i \in I} \subset \mathcal{C}\left(\mathbb{R}^{n}\right), B \in \mathcal{B}\left(\mathbb{R}^{n}\right)$ and $A=\bigcap_{i \in I}\left(B+A_{i}\right)$. Then the following statements are equivalent:

(a) $\inf _{i \in I}\left[A_{i},\{0\}\right]=[A, B]$ (infimum in MRH cone).

(b) $\bigcap_{i \in I}\left(D+B+A_{i}\right)=D+A$ for all $D \in \mathcal{B}\left(\mathbb{R}^{n}\right)$.

Corollary 4.3 Let $\left\{A_{i}\right\}_{i \in I} \subset \mathcal{C}\left(\mathbb{R}^{n}\right)$ and $A=\bigcap_{i \in I} A_{i}$. Then the following statements are equivalent:

(a) $\inf _{i \in I}\left[A_{i},\{0\}\right]=[A,\{0\}]$ (infimum in MRH cone).

(b) $\bigcap_{i \in I}\left(D+A_{i}\right)=D+A$ for all $D \in \mathcal{B}\left(\mathbb{R}^{n}\right)$ (translation property of intersection with respect to $\left.\mathcal{B}\left(\mathbb{R}^{n}\right)\right)$.

Example 4.4 Let $A_{t}=(0,0) \vee(t, 1) \subset \mathbb{R}^{2}$. The family $\left\{A_{t}\right\}_{t \in \mathbb{R}}$ (Fig. 1) is an exhauster of the function $h$ defined by

$$
h\left(x_{1}, x_{2}\right)=\left\{\begin{array}{llll}
x_{2} & \text { if } x_{1}=0 & \text { and } & x_{2}>0 \\
0 & \text { if } x_{1} \neq 0 & \text { or } & x_{2} \leqslant 0
\end{array}\right.
$$

The function $h$ is not continuous but it is upper semicontinuous. Let $H$ be the closed lower halfplane $x_{2} \leqslant 0$. Then $\bigcap_{t \in \mathbb{R}}\left(H+A_{t}\right)=H+\{(0,1)\} \neq H+\{(0,0)\}=H+\bigcap_{t \in \mathbb{R}} A_{t}$. The exhauster $E^{*}(h)=\left\{A_{t}\right\}_{t \in \mathbb{R}}$ does not have the translation property of intersection with respect to $\mathcal{C}\left(\mathbb{R}^{2}\right)$. On the other hand let $D \in \mathcal{B}\left(\mathbb{R}^{2}\right)$. If $x \in \bigcup_{t \in \mathbb{R}}\left(D+A_{t}\right)$ then $(x-D) \cap A_{t} \neq \emptyset$ for all $t$. Since $x-D$ is compact, the set $x-D$ intersects both $\mathbb{R}_{+} \times\{0\}$ and $\mathbb{R}_{-} \times\{0\}$. Since $D$ is convex, we have $0=(0,0) \in x-D$, and $x \in D$. Therefore, the exhauster $E^{*}(h)=\left\{A_{t}\right\}_{t \in \mathbb{R}}$ does have the translation property of intersection with respect to $\mathcal{B}\left(\mathbb{R}^{2}\right)$. 
Fig. 2 The family of segments

$\left\{A_{t}\right\}_{t \in[0, \pi)}$ in Example 5.3

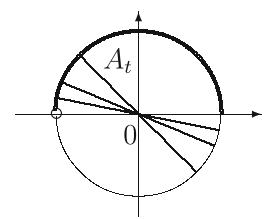

\section{Shadowing property}

The shadowing property, mentioned in Theorem 3.1 (b), is a generalization of separation of two sets by another (separating) set. A shadowing set shadows, in general, arbitrary number of sets or a family of sets. The notion of shadowing was introduced in [15].

Theorem 5.1 (shadowing property of intersection) Let $\left\{A_{i}\right\}_{i \in I} \subset \mathcal{C}\left(\mathbb{R}^{n}\right)$ and $A=\bigcap_{i \in I} A_{i}$. Then the following statements are equivalent:

(a) $\inf _{i \in I} p_{A_{i}}=p_{A}$.

(b) For all $\left\{a_{i}\right\}_{i \in I} \in \prod_{i \in I} A_{i}$ and $\varepsilon>0$ we have $(A+\varepsilon \mathbb{B}) \cap \bigvee_{i \in I} a_{i} \neq \emptyset$ (generalized shadowing property).

Proof (a) $\Rightarrow$ (b) Let us assume that (b) does not hold. Then there exist $\left\{a_{i}\right\}_{i \in I} \in \prod_{i \in I} A_{i}$ and $\varepsilon>0$ such that $(A+\varepsilon \mathbb{B}) \cap \bigvee_{i \in I} a_{i}=\emptyset$. Hence the convex sets $(A+\varepsilon \mathbb{B})$ and $\bigvee_{i \in I} a_{i}$ can be weakly separated by a hyperplane. Then $A$ and $\bigvee_{i \in I} a_{i}$ can be strictly separated. There exist $x \in \mathbb{R}^{n}$ and $\alpha, \beta \in \mathbb{R}$ such that $\langle a, x\rangle \leqslant \alpha<\beta \leqslant\left\langle a_{i}, x\right\rangle$ for all $i \in I$ and all $a \in A$. Therefore, $p_{A}<\inf _{i \in I} p_{A_{i}}$, and (a) does not hold.

(b) $\Rightarrow$ (a) Let us assume that (a) does not hold. Then $p_{A}(x) \leqslant \alpha<\beta \leqslant \inf _{i \in I} p_{A_{i}}(x)$ for some $x \in \mathbb{R}^{n}$ and $\alpha, \beta \in \mathbb{R}$. For each $i \in I$ let us choose $a_{i} \in A_{i}$ such that $\beta \leqslant\left\langle a_{i}, x\right\rangle$. Then the sets $A$ and $\bigvee_{i \in I} a_{i}$ are strictly separated by two parallel hyperlanes $\langle\cdot, x\rangle=\alpha$ and $\langle\cdot, x\rangle=\beta$. Hence the condition (b) does not hold.

Corollary 5.2 Let $\left\{A_{i}\right\}_{i \in I} \subset \mathcal{B}\left(\mathbb{R}^{n}\right)$ and $A=\bigcap_{i \in I} A_{i}$. Then the following statements are equivalent:

(a) $\inf _{i \in I} p_{A_{i}}=p_{A}$.

(b) For all $\left\{a_{i}\right\} \in \prod_{i \in I} A_{i}$ we have $A \cap \bigvee_{i \in I} a_{i} \neq \emptyset$ (shadowing property).

Proof In this corollary the set $A$ is compact and convex. Hence $A$ does not intersect closed convex sets if and only if $A$ can be strictly separated from that set. Then the corollary follows from Theorem 5.1.

The following example shows that the closedness of the set $\bigvee_{i \in I} a_{i}$ in the statement (b) of Corollary 5.2 is essential.

Example 5.3 Let us consider an upper exhauster $\left\{A_{t}\right\}_{t \in[0, \pi)}$, where $A_{t}=(\cos t, \sin t) \vee$ $(-\cos t,-\sin t) \subset \mathbb{R}^{2}$ (Fig. 2).

We have $\inf _{t \in[0, \pi)} p_{A_{t}}=0$. Notice that $A=\bigcap_{t \in[0, \pi)} A_{t}=\{0\}$. Denote $a_{t}=$ $(\cos t, \sin t)$. Then $0 \in \overline{\operatorname{conv}}\left\{a_{t} \mid t \in[0, \pi)\right\}=\bigvee_{t \in[0, \pi)} a_{t}$, but $0 \notin \operatorname{conv}\left\{a_{t} \mid t \in[0, \pi)\right\}$. Hence $A \cap \bigvee_{i \in I} a_{i} \neq \emptyset$, but $A \cap \operatorname{conv}\left\{a_{t} \mid t \in[0, \pi)\right\}=\emptyset$.

\section{Reducing infinite exhausters to single sets}

The ability of replacing a given exhauster with one convex set is applicable also to many cases of exhausters irreducible to one convex set. Indeed, if an exhauster $\left\{A_{i}\right\}_{i \in I}$, is a union 
of a number of exhausters $\left\{A_{i}\right\}_{i \in I_{\lambda}}, \lambda \in \Lambda$, where $I=\bigcup_{\lambda \in \Lambda} I_{\lambda}$ and if each of exhausters $\left\{A_{i}\right\}_{i \in I_{\lambda}}$ is reducible to one set $B_{\lambda}$ then the exhauster $\left\{A_{i}\right\}_{i \in I_{\lambda}}$ can be reduced to "smaller" exhauster $\left\{B_{\lambda}\right\}_{\lambda \in \Lambda}$. Also if for a given convex set $C$ the exhauster $\left\{C \dot{+} A_{i}\right\}_{i \in I}$ is reducible to one convex set $B$ then the exhauster $\left\{A_{i}\right\}_{i \in I}$ is reducible to the pair of sets $(B, C)$ in the sense that $\inf _{i \in I} p_{A_{i}}=p_{B}-p_{C}$.

The following theorem connects reducibility to one set with the translation property of intersection.

Theorem 6.1 (on reducing infinite upper exhausters to one set) Let $\left\{A_{i}\right\}_{i \in I} \subset \mathcal{C}\left(\mathbb{R}^{n}\right)$ and $A=\bigcap_{i \in I} A_{i}$. Then the following statements are equivalent:

(a) $\inf _{i \in I} p_{A_{i}}=p_{A}$.

(b) For all $D \in \mathcal{C}\left(\mathbb{R}^{n}\right)$ we have $\bigcap_{i \in I}\left(D \dot{+} A_{i}\right)=D \dot{+} A$ (translation property of intersection with respect to $\left.\mathcal{C}\left(\mathbb{R}^{n}\right)\right)$.

Proof (a) $\Rightarrow$ (b) Let us assume that (b) does not hold. Then there exist $D \in \mathcal{C}\left(\mathbb{R}^{n}\right)$ and $x \in \bigcap_{i \in I}\left(D \dot{+} A_{i}\right) \backslash(D \dot{+} A)$. Since $x$ can be strictly separated from $D \dot{+} A$, there exists $y \in \mathbb{R}^{n}$ such that

$$
p_{D}(y)+p_{A}(y)=p_{D \dot{+} A}(y)<\langle x, y\rangle \leqslant \inf _{i \in I} p_{D \dot{+} A_{i}}(y)=p_{D}(y)+\inf _{i \in I} p_{A_{i}}(y) .
$$

Hence (a) does not hold.

(b) $\Rightarrow$ (a) Let us assume that (a) does not hold. Then $p_{A}(x) \leqslant \alpha<\beta \leqslant \inf _{i \in I} p_{A_{i}}(x)$ for some $x \in \mathbb{R}^{n}$ and $\alpha, \beta \in \mathbb{R}$. By $H_{\leqslant \alpha}$ we denote the halfspace $\left\{y \in \mathbb{R}^{n} \mid\langle y, x\rangle \leqslant \alpha\right\}$. Denote $D=H_{\leqslant 0}$. We have $D \dot{+} A \subset H_{\leqslant \alpha}$. On the other hand $H_{\leqslant \beta} \subset D \dot{+} A_{i}$ for all $i \in I$. Hence $D \dot{+} A \varsubsetneqq \bigcap_{i \in I}\left(D \dot{+} A_{i}\right)$, and the condition (b) does not hold.

Corollary 6.2 Let the family $\left\{A_{i}\right\}_{i \in I} \subset \mathcal{B}\left(\mathbb{R}^{n}\right)$ have bounded union $\bigcup_{i \in I} A_{i}$ and $A=$ $\bigcap_{i \in I} A_{i}$. Then the following statements are equivalent:

(a) $\inf _{i \in I} p_{A_{i}}=p_{A}$.

(b) For all $D \in \mathcal{B}\left(\mathbb{R}^{n}\right)$ we have $\bigcap_{i \in I}\left(D+A_{i}\right)=D+A$ (translation property of intersection with respect to $\mathcal{B}\left(\mathbb{R}^{n}\right)$ ).

Proof By Theorem 6.1 the condition (b) follows from (a). Let us assume that (a) does not hold. Then $p_{A}(x) \leqslant \alpha<\beta \leqslant \inf _{i \in I} p_{A_{i}}(x)$ for some $x \in \mathbb{R}^{n}$ and $\alpha, \beta \in \mathbb{R}$. Hence there exists $\left\{a_{i}\right\}_{i \in I} \in \prod_{i \in I} A_{i}$ such that $\left\langle a_{i}, x\right\rangle=\beta$. For all $y \in \bigvee_{i \in I} a_{i}$ we have $\langle y, x\rangle=\beta$. Hence $A$ and $\bigvee_{i \in I} a_{i}$ do not intersect. Denote $D=-\bigvee_{i \in I} a_{i}$. The vector 0 does not belong to $A+D$. On the other hand $0 \in D+A_{i}$ for all $i \in I$. Then $D+A \varsubsetneqq \bigcap_{i \in I}\left(D+A_{i}\right)$, and since $\bigvee_{i \in I} a_{i} \subset \bigvee_{i \in I} A_{i}$ is bounded, the condition (b) does not hold.

\section{Minimality of upper exhausters}

We say that an upper exhauster $E^{*}(h)=\left\{A_{i}\right\}_{i \in I} \subset \mathcal{B}\left(\mathbb{R}^{n}\right)$ is inclusion-minimal (or minimal in the sense of Demyanov [3]) if $\inf _{i \in I \backslash\{k\}} p_{A_{i}} \neq \inf _{i \in I} p_{A_{i}}=h$ for all $k \in I$. It means that if we drop any element of the exhauster we obtain an exhauster of different function $h$.

An exhauster $E^{*}(h)$ is $I$-minimal if $\inf \left(p_{A_{k}^{\prime}}, \inf _{i \in I \backslash\{k\}} p_{A_{i}}\right) \neq \inf _{i \in I} p_{A_{i}}=h$ for all $k \in I$ and all $\mathcal{B}\left(\mathbb{R}^{n}\right) \ni A_{k}^{\prime} \varsubsetneqq A_{k}$. It means that if we replace any element of the exhauster with its proper subset we obtain an exhauster of a different function $h$.

We call an upper exhauster $E^{*}(h)=\left\{A_{i}\right\}_{i \in I}$ minimal if it is inclusion-minimal and I-minimal. 
Fig. $3 h=\inf \left(p_{A_{1}}, p_{A_{2}}\right)=$ $\inf \left(p_{B_{1}}, p_{B_{2}}\right)$
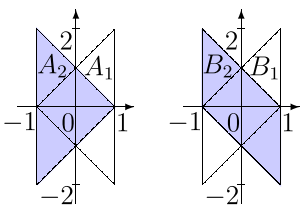

Fig. $4 h=\inf \left(p_{A_{1}}, p_{A_{2}}\right)=$ $\inf \left(p_{A_{1}}, p_{B_{1}}, p_{C_{x}}\right)$
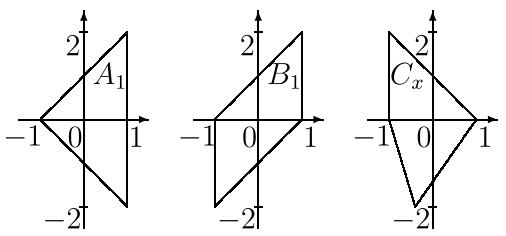

In Theorem 7.1 we prove that an upper exhauster $E^{*}(h)$ is minimal if and only if it is minimal in the sense of Demyanov-Roshchina, i.e. if there exists no other upper exhauster $\tilde{E}^{*}(h)$, satisfying the following property:

For every $\tilde{C} \in \tilde{E}^{*}(h)$, there exists $C \in E^{*}(h)$ such that $\tilde{C} \subset C$ [28]. In other words the exhauster $\tilde{E}^{*}(h)$ is finer than $E^{*}(h)$.

Theorem 7.1 (on minimality) An exhauster $E^{*}(h)=\left\{A_{i}\right\}_{i \in I} \subset \mathcal{B}\left(\mathbb{R}^{n}\right)$ is minimal in the sense of Demyanov-Roshchina if and only if it is inclusion-minimal and I-minimal.

Proof If an exhauster $E^{*}(h)$ is minimal in the sense of Demyanov-Roshchina, then obviously it is minimal by inclusion and $I$-minimal.

Conversely, let $\tilde{E}^{*}(h)=\left\{A_{j}^{\prime}\right\}_{j \in J}$ and $\tilde{E}^{*}(h)$ be finer than $E^{*}(h)$. Given arbitrary $A_{l}^{\prime}$, there exists $A_{k} \in E^{*}(h)$ such that $A_{l}^{\prime} \subset A_{k}$. We have

$$
\begin{aligned}
h= & \inf _{j \in J} p_{A_{j}^{\prime}}=\min \left(\inf _{j \in J} p_{A_{j}^{\prime}}, p_{A_{l}^{\prime}}\right)=\min \left(h, p_{A_{l}^{\prime}}\right)=\min \left(\inf _{i \in I} p_{A_{i}}, p_{A_{l}^{\prime}}\right) \\
& \leqslant \min \left(\inf _{i \in I \backslash\{k\}} p_{A_{i}}, p_{A_{l}^{\prime}}\right) \leqslant \min \left(\inf _{i \in I \backslash\{k\}} p_{A_{i}}, p_{A_{k}}\right)=\inf _{i \in I} p_{A_{i}}=h .
\end{aligned}
$$

Then the family $\hat{E}^{*}=\left\{A_{i}\right\}_{i \in I \backslash\{k\}} \cup\left\{A_{l}^{\prime}\right\}$ is also an upper exhauster of $h$, i.e. $\hat{E}^{*}=\hat{E}^{*}(h)$. Since the exhauster $E^{*}(h)$ is $I$-minimal, we have $\hat{E}^{*}=E^{*}(h)$. Hence $A_{k}=A_{l}^{\prime}$, and $\tilde{E}^{*}(h) \subset E^{*}(h)$. But the exhauster $E^{*}(h)$ is inclusion-minimal, hence $\tilde{E}^{*}(h)=E^{*}(h)$.

Example 7.2 We present different minimal exhausters of the same function $h$. Consider two triangles $A_{1}=(1,2) \vee(1,-2) \vee(-1,0)$ and $A_{2}=(-1,2) \vee(-1,-2) \vee(0,1)$ in $\mathcal{B}\left(\mathbb{R}^{2}\right)$. Let $h=\min \left(p_{A_{1}}, p_{A_{2}}\right)$. Then $E^{*}(h)=\left\{A_{1}, A_{2}\right\}$ is an upper exhauster of the function $h$. Moreover the upper exhauster $E^{*}(h)$ is minimal. On the other hand, the upper exhauster $E_{1}^{*}(h)=\left\{B_{1}, B_{2}\right\}$ is also minimal. Then the uniqueness of minimal exhausters does not hold (Fig. 3). These two exhausters were already presented in [15].

Let $C_{x}=(1,0) \vee(x,-2) \vee(-1,0) \vee(-1,2)$, where $-1<x<1$. Then $E_{x}^{*}(h)=$ $\left\{A_{1}, B_{1}, C_{x}\right\}$ is one of a continuum of three elements minimal exhausters (see Fig. 4).

Let $C_{w}^{1}=(1,0) \vee(-1,-2) \vee(-1,0) \vee(w, 2), C_{x}^{2}=(1,0) \vee(1,-2) \vee(-1,0) \vee(x, 2)$, $C_{y}^{3}=(1,0) \vee(y,-2) \vee(-1,0) \vee(-1,2)$ and $C_{z}^{4}=(1,0) \vee(z,-2) \vee(-1,0) \vee(-1,2)$, where $w, x, y, z \in(-1,1)$. Then $E_{w x y z}^{*}(h)=\left\{C_{w}^{1}, C_{x}^{2}, C_{y}^{3}, C_{z}^{4}\right\}$ is one of a continuum of four elements minimal exhausters (see Fig. 5). There is no $n$-elements minimal exhausters of $h$, where $n \geqslant 5$. 
Fig. $5 h=\inf \left(p_{A_{1}}, p_{A_{2}}\right)=$ $\inf \left(p_{C_{w}^{1}}, p_{C_{x}^{2}}, p_{C_{y}^{3}}, p_{C_{z}^{4}}\right)$
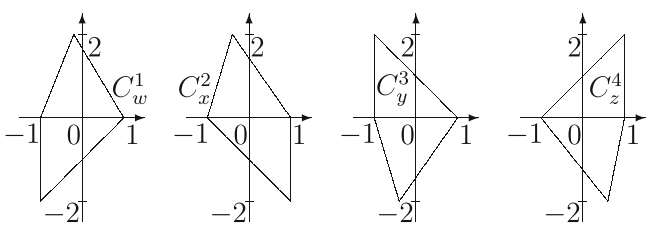

Fig. 6 An infinite minimal exhauster

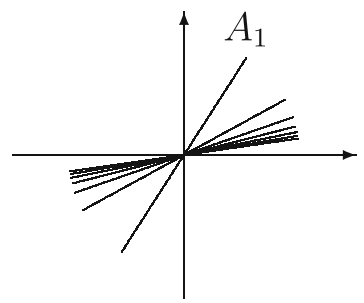

Example 7.3 In this example, we present a minimal infinite exhauster $E^{*}(h)=\left\{A_{n}\right\}_{n \in \mathbb{N}}$, where $A_{n}=\left(\cos \frac{i}{n}, \sin \frac{i}{n}\right) \vee\left(-\cos \frac{i}{n},-\sin \frac{i}{n}\right) \subset \mathbb{R}^{2}$ and $h=\inf _{n \in \mathbb{N}} p_{A_{n}}$ (see Fig. 6).

Notice that for any $k \in \mathbb{N}$ we have

$$
\inf _{n \in \mathbb{N} \backslash\{k\}} p_{A_{n}}\left(-\sin \frac{i}{n}, \cos \frac{i}{n}\right)>h\left(-\sin \frac{i}{n}, \cos \frac{i}{n}\right)=0 .
$$

Hence our exhauster $E^{*}(h)$ is inclusion-minimal. On the other hand for any $k \in \mathbb{N}$ if we replace the segment $A_{k}$ with its proper subsegment $A_{k}^{\prime}$ then there exists a small real number $x$ (positive or negative) such that

$$
\min \left(p_{A_{k}^{\prime}}, \inf _{n \in \mathbb{N} \backslash\{k\}} p_{A_{n}}\right)\left(-\sin \frac{i}{n}+x, \cos \frac{i}{n}\right)<h\left(-\sin \frac{i}{n}+x, \cos \frac{i}{n}\right) .
$$

Hence the exhauster $E^{*}(h)$ is $I(=\mathbb{N})$-minimal.

\section{Reducing exhausters with the help of polars of convex sets}

Let $\mathcal{C}_{0}\left(\mathbb{R}^{n}\right)$ be a family of sets from $\mathcal{C}\left(\mathbb{R}^{n}\right)$ containing 0 . Let $A \in \mathcal{C}_{0}\left(\mathbb{R}^{n}\right)$. By $A^{\circ}$ we denote the set $\left\{x \in \mathbb{R}^{n} \mid p_{A}(x) \leqslant 1\right\}$ and call it the polar set or polar of $A$.

Let $\mathcal{R}$ be the family of all radiant subsets $B$ of $\mathbb{R}^{n}$, that is $[0,1] \cdot B \subset B$ and the intersection of $B$ and any ray $L$ with initial point 0 is closed. We call a family $\left\{B_{i}\right\}_{i \in I} \subset \mathcal{C}\left(\mathbb{R}^{n}\right)$ a convex covering of $B \in \mathcal{R}$ if $[0,1) \cdot B \subset \bigcup_{i \in I} B_{i} \subset B$. We call a convex covering $\left\{B_{i}\right\}_{i \in I}$ of $B$ inclusion-minimal if $\left\{B_{i}\right\}_{i \in I \backslash\{k\}}$ is no longer a convex covering of $B$ for all $k \in I$. It is $I$ minimal if $\left\{B_{i}\right\}_{i \in I \backslash\{k\}} \cup\{C\}$ is not a convex covering of $B$ for all $k \in I$ and all $C \in \mathcal{C}\left(\mathbb{R}^{n}\right)$ such that $B_{k} \varsubsetneqq C$. We call the covering $\left\{B_{i}\right\}_{i \in I}$ minimal if it is inclusion-minimal and $I$-minimal.

Theorem 8.1 Let $\left\{A_{i}\right\}_{i \in I} \subset \mathcal{C}\left(\mathbb{R}^{n}\right)$, a function $h: \mathbb{R}^{n} \longrightarrow \mathbb{R}$ be p.h. and non-negative, and $B_{h}=\left\{x \in \mathbb{R}^{n} \mid h(x) \leqslant 1\right\}$. Then $E^{*}(h)=\left\{A_{i}\right\}_{i \in I}\left(h=\inf _{i \in I} p_{A_{i}}\right)$ if and only if the family $\left\{A_{i}^{\circ}\right\}_{i \in I}$ is a convex covering of $B_{h}$.

Proof $\Rightarrow$ ) Let $x \in[0,1) \cdot B_{h}$. Then $\inf _{i \in I} p_{A_{i}}(x)=h(x)<1$. Hence $p_{A_{k}}(x)<1$ for some $k \in I$. Therefore, $x \in A_{k}^{\circ} \subset \bigcup_{i \in I} A_{i}^{\circ}$. On the other hand, if $x \in A_{k}^{\circ}$ for some $k \in I$ then $h(x)=\inf _{i \in I} p_{A_{i}}(x) \leqslant p_{A_{k}}(x) \leqslant 1$. Hence $x \in B_{h}$. 

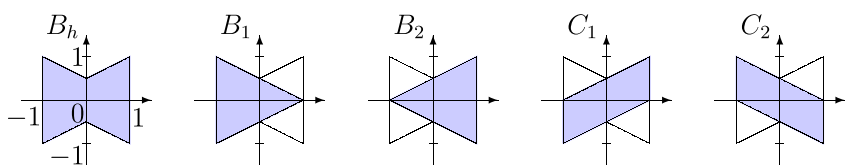

Fig. $7 B_{h}=B_{1} \cup B_{2}=C_{1} \cup C_{2}$

Fig. $8 B_{h}=D_{1} \cup D_{2} \cup D_{3} \cup D_{4}$
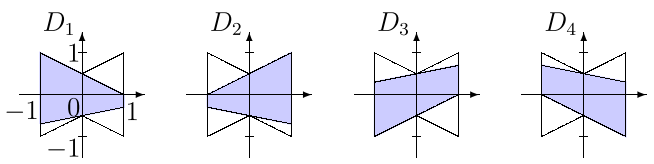

Fig. $9 \quad B=B_{1} \cup B_{2} \cup B_{3}$
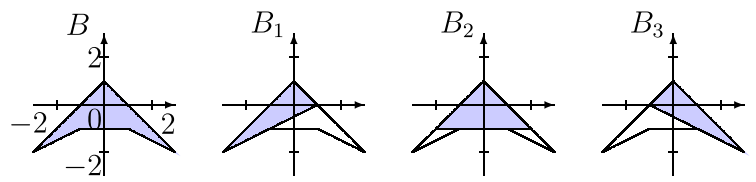

Fig. 10 Polars of $B_{1}, B_{2}, B_{3}$ and the union of all maximal convex subsets of $B$
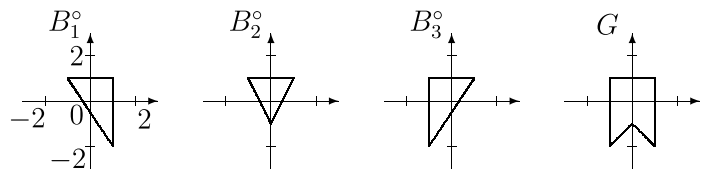

$\Leftarrow)$ Since $\bigcup_{i \in I} A_{i}^{\circ} \subset B_{h}$, we have $A_{i}^{\circ} \subset B_{h}$ for all $i$. Then $h \leqslant p_{A_{i}}$. Hence $h \leqslant$ $\inf _{i \in I} p_{A_{i}}$. Let $x \in \mathbb{R}^{n}$ and $\varepsilon>0$. If $h(x)>0$ then $\frac{x}{(1+\varepsilon) h(x)} \in[0,1) \cdot B_{h} \subset \bigcup_{i \in I} A_{i}^{\circ}$. Hence there exists $k \in I$ such that $\frac{x}{(1+\varepsilon) h(x)} \in A_{k}^{\circ}$, and $p_{A_{k}}\left(\frac{x}{(1+\varepsilon) h(x)}\right) \leqslant 1$. Then $p_{A_{k}}(x) \leqslant$ $(1+\varepsilon) h(x)$, and $h(x)=\inf _{i \in I} p_{A_{i}}$. If $h(x)=0$ then $[0, \infty) \cdot x \subset B_{h}$. For any $M>0$ there exist $i_{M} \in I$ such that $M x \in A_{i_{M}}^{\circ}$. Then $p_{A_{i_{M}}}(M x) \leqslant 1$, and $p_{A_{i_{M}}}(x) \leqslant \frac{1}{M}$. Hence $\inf _{i \in I} p_{A_{i}}(x)=0$.

Corollary 8.2 In Theorem 8.1 the exhauster $E^{*}(h)=\left\{A_{i}\right\}_{i \in I}$ is minimal if and only if the convex covering $\left\{A_{i}^{\circ}\right\}_{i \in I}$ of $B_{h}$ is minimal.

Example 8.3 We present two p.h. functions and their minimal exhausters. The figures show how easily we can geometrically construct minimal exhausters of some functions. First we consider a function $h\left(x_{1}, x_{2}\right)=\min \left(\max \left(x_{1}+2 x_{2}, x_{1}-2 x_{2},-x_{1}\right), \max \left(-x_{1}+2 x_{2},-x_{1}-\right.\right.$ $\left.2 x_{2}, x_{1}\right)$ ). In Figs. 7 and 8 we present the set $B_{h}=\left\{x \in \mathbb{R}^{2} \mid h(x) \leqslant 1\right\}$ and different minimal convex coverings $\left\{B_{1}, B_{2}\right\},\left\{C_{1}, C_{2}\right\}$ and $\left\{D_{1}, D_{2}, D_{3}, D_{4}\right\}$ of the radiant set $B_{h}$. By Corollary 8.2 families $\left\{B_{1}^{\circ}, B_{2}^{\circ}\right\},\left\{C_{1}^{\circ}, C_{2}^{\circ}\right\}$ and $\left\{D_{1}^{\circ}, D_{2}^{\circ}, D_{3}^{\circ}, D_{4}^{\circ}\right\}$ are different minimal exhausters of $h$.

Figure 9 shows a radiant set $B$ and its unique minimal convex covering $\left\{B_{1}, B_{2}, B_{3}\right\}$. If $h$ is a gauge function of $B$ (in equivalent way $B=B_{h}$ ) then $\left\{B_{1}^{\circ}, B_{2}^{\circ}, B_{3}^{\circ}\right\}$ (see Fig. 10) is a unique minimal exhauster of $h$.

In Fig. 10 the set $G$ is a union of polars of all maximal convex subsets of $B$. By the way a convex hull of $G$ is equal to Clarke subdifferential [2] of the function $h$ at origin.

Acknowledgments The authors wish to express their appreciation to an anonymous referee for valuable hints and comments. The authors also thank İlknur Atasever Güvenç, Didem Tozkan and Mustafa Soyertem for careful reading of the paper and their remarks. 
Open Access This article is distributed under the terms of the Creative Commons Attribution License which permits any use, distribution, and reproduction in any medium, provided the original author(s) and the source are credited.

\section{References}

1. Caprari, E., Penot, J.P.: Tangentially ds functions. Optimization 56, 13-29 (2007)

2. Clarke, F.H.: Optimization and nonsmooth analysis. J. Wiley Pub. Comp., New York (1983)

3. Demyanov, V.F.: Exhausters and convexificators-new tools in nonsmooth analysis. In: Demyanov, V.F., Rubinov, A.M. (eds.) Quasidifferentiability and Related Topics, pp. 85-137. Kluwer Academic Publishers, Dortrecht (2000)

4. Demyanov, V.F., Roshchina, V.: Optimality conditions in term of upper and lower exhausters. Optimization 55, 525-540 (2006)

5. Demyanov, V.F., Roshchina, V.: Exhausters and subdifferentials in nonsmooth analysis. Optimization 57, $41-56(2008)$

6. Demyanov, V.F., Roshchina, V.: Exhausters, optimality conditions and related problems. J. Global Optim. 40, 71-85 (2008)

7. Demyanov, V.F., Rubinov, A.M.: On quasidifferentiable functionals. Doklady USSR Acad. Sci. 250(1), $21-25(1980)$

8. Demyanov, V.F., Rubinov, A.M.: On some approaches to the nonsmooth optimization problems. Econ. Math. Method 17(6), 1153-1174 (1981) (in Russain)

9. Demyanov, V.F., Rubinov, A.M.: Elements of quasidifferential calculus. In: Nonsmooth Problems of Optimization Theory and Control, Vol. 1 . Leningrad University Press, Leningrad (1982)

10. Demyanov, V.F., Rubinov, A.M.: Quasidifferential calculus, optimization software Inc. Springer, New York (1986)

11. Drewnowski, L.: Additive and countably additive correspondences. Comment. Math. 19, 25-54 (1976)

12. Glover, B.M., Ishizuka, Y., Jeyakumar, V., Tuan, H.D.: Complete characterizations of global optimality for problems involving the pointwise minimum of sublinear functions. SIAM J. Optim. 6(2), 362-372 (1996)

13. Grzybowski, J.: Minimal pairs of compact sets. Arch. Math. 63, 173-181 (1994)

14. Grzybowski, J., Küçük, M., Küçük, Y., Urbański, R.: Minkowski-Rådström-Hörmander cone. Pac. J. Optim. (to appear) (2014)

15. Grzybowski, J., Pallaschke, D., Urbański, R.: Reduction of finite exhausters. J. Glob. Optim. 46, 589-601 (2010)

16. Hörmander, L.: Sur la fonction d'appui des ensembles convexes dans un espace localement convexe. Arkiv Math. 3, 181-186 (1954)

17. Hukuhara, M.: Intégration des Applications measurables dont la valuer est un compact convexe. Func. Ekvacioj. 10, 205-223 (1967)

18. Markow, S.: On the algebraic properties of convex bodies and some applications. J. Convex Anal. 7, 129-166 (2000)

19. Pallaschke, D., Scholtes, S., Urbański, R.: On minimal pairs of convex compact sets. Bull. Polish Acad. Sci. Math. 39, 1-5 (1991)

20. Pallaschke, D., Urbański, R.: Pairs of Compact Convex Sets, Fractional Arithmetic with Convex Sets, Mathematics and Its Applications. Kluwer Academic Publisher, Dortrecht (2002)

21. Pontryagin, L.S.: On linear differential games II. Dokl. Acad. Nauk SSSR 175, 764-766 (1967)

22. Praksash, P., Sertel, R.: Hyperspaces of topological vector spaces: their embedding in topological vector spaces. Proc. Am. Math. Soc. 61, 163-168 (1976)

23. Pshenichnyi, B.N.: Necessary Conditions for an Extremum. Marcel Dekker, New York (1971)

24. Pshenichnyi, B.N.: Convex Analysis and Extremal Problems. Nauka Publishers, Moscow (1980)

25. Rabinovich, M.G.: An embedding theorem for space of convex sets. Sib. Math. J. 8, 275-279 (1967)

26. Rådström, H.: An embedding theorem for spaces of convex sets. Proc. Am. Math. Soc. 3, 165-169 (1952)

27. Rockafellar, R.T.: Convex Analysis. Princeton University Press, Princeton (1970)

28. Roshchina, V.: Reducing exausters. J. Optim. Theory Appl. 136, 261-273 (2008)

29. Roshchina, V.: On conditions for the minimality of exausters. J. Convex Anal. 15(4), 859-868 (2008)

30. Schmidt, K.: Embedding theorems for classes of convex sets. Acta Appl. Math. 5, 209-223 (1986)

31. Urbański, R.: A generalization of the Minkowski-Rådström-Hörmander theorem. Bull. Acad. Polon. Sci. Sér. Sci. Math. Astronom. Phys. 24, 709-715 (1976) 